J. Appl. Numer. Optim. 1 (2019), No. 1, pp. 1-12

Available online at http://jano.biemdas.com

https://doi.org/10.23952/jano.1.2019.1.01

\title{
APPROXIMATION OF COMMON SOLUTIONS OF A SPLIT INCLUSION PROBLEM AND A FIXED-POINT PROBLEM
}

\author{
LU-CHUAN CENG \\ Department of Mathematics, Shanghai Normal University, Shanghai 200234, China
}

\begin{abstract}
The purpose of this paper is to introduce a composite viscosity iterative algorithm for finding a common solution of a split variational inclusion problem and a fixed point of a family of pseudocontractive mappings. Strong convergence of the proposed iterative algorithm is obtained under some mild assumptions in the framework of Hilbert spaces.

Keywords. Split variational inclusion problem; Fixed-point problem; Pseudocontractive mapping; Composite viscosity iterative method.
\end{abstract}

2010 Mathematics Subject Classification. 47H09, 47J20.

\section{INTRODUCTION-PRELIMINARIES}

Let $H_{1}$ and $H_{2}$ be two real Hilbert spaces with inner product $\langle\cdot, \cdot\rangle$ and norm $\|\cdot\|$, while $H$ refers to as any of these spaces. Let $C$ be a nonempty closed convex subset of $H_{1}$, and let $Q$ be a nonempty closed convex subset of $H_{2}$. In 1994, Censor and Elfving [7] introduced the following well known split feasibility problem: find $x \in H_{1}$ such that

$$
x \in C, \quad A x \in Q,
$$

where $A: H_{1} \rightarrow H_{2}$ is a bounded linear operator. The split feasibility problem has attracted many authors' attention due to its application in signal processing and image reconstruction, with particular progress in intensity-modulated radiation therapy $[1,8,9,20]$ and many other applied fields.

In 2002, Byrne [1] introduced the following so-called CQ algorithm.

$$
x_{0} \in H_{1}, x_{n+1}=P_{C}\left(I-\gamma A^{*}\left(I-P_{Q}\right) A\right) x_{n}, \quad n \geq 1,
$$

where $A^{*}$ is the adjoint operator of $A, \gamma>0$ is a real constant in $\left(0, \frac{2}{r}\right)$ ( $r$ is the spectral radius of the self-adjoint operator $\left.A^{*} A\right), P_{C}$ is the metric projection from $H_{1}$ onto $C, P_{Q}$ is the metric projection from $H_{2}$ onto $Q$ and $I$ is the identity mapping on $H_{1}$. He proved that the sequence $\left\{x_{n}\right\}$ generated in the CQ algorithm converges weakly to some solution of the split feasibility problem. Recently, many authors intensively investigated the split feasibility problem based on different regularization methods in Hilbert spaces or Banach spaces; see $[2,3,4,5,6,18,21]$ and the references therein.

Let $B: C \rightarrow H$ be a nonlinear mapping. The classical variational inequality (VI) is to find $x^{*} \in C$ such that

$$
\left\langle B x^{*}, x-x^{*}\right\rangle \geq 0, \quad \forall x \in C
$$

E-mail address: zenglc@ hotmail.com.

Received November 30, 2019; Accepted February 1, 2019.

(C)2019 Journal of Applied and Numerical Optimization 
We denote by $\operatorname{VI}(C, B)$ the solution set of VI (1.1). As a very effective and powerful tool, VI (1.1) has been applied to study a wide range of problems arising in differential equations, mechanics, contact problems in elasticity, optimization and control problems, management science, operations research, and general equilibrium problems in economics and transportation.

A set-valued mapping $M: H \rightarrow 2^{H}$ is said to be monotone if, for all $x, y \in H, f \in M x$ and $g \in M y$ imply $\langle x-y, f-g\rangle \geq 0$. A monotone mapping $M: H \rightarrow 2^{H}$ is maximal if the $\operatorname{graph} \operatorname{Gph}(M)$ of $M$ is not properly contained in the graph of any other monotone mapping. It is known that a monotone mapping $M$ is maximal if and only if for $(x, f) \in H \times H,\langle x-y, f-g\rangle \geq 0, \forall(y, g) \in \operatorname{Gph}(M)$ implies $f \in M x$. Let $M: H \rightarrow 2^{H}$ be a multi-valued maximal monotone mapping. For any positive number $\lambda$ and identity operator $I$ on $H$, the single-valued mapping $J_{\lambda}^{M}: H \rightarrow H$ defined by

$$
J_{\lambda}^{M}(x):=(I+\lambda M)^{-1}(x), \quad \forall x \in H,
$$

is called the resolvent operator associated with $M$. It is known that the resolvent operator $J_{\lambda}^{M}$ is firmly nonexpansive, in particular, nonexpansive.

Let $B_{1}: H_{1} \rightarrow 2^{H_{1}}$ and $B_{2}: H_{2} \rightarrow 2^{H_{2}}$ be multi-valued maximal monotone mappings. We consider the following split variational inclusion problem (SVIP): find $x^{*} \in H_{1}$ such that

$$
0 \in B_{1}\left(x^{*}\right),
$$

and

$$
y^{*}=A x^{*} \in H_{2} \quad \text { solves } \quad 0 \in B_{2}\left(y^{*}\right) .
$$

In this paper, $\operatorname{Sol}\left(B_{1}, B_{2}\right)$ stands for the solution set of SVIP (1.2)-(1.3).

It is obvious that SVIP (1.2)-(1.3) is equivalent to the problem of finding $x^{*} \in H_{1}$ with $x^{*}=J_{\lambda}^{B_{1}}\left(x^{*}\right)$ such that

$$
y^{*}=A x^{*} \in H_{2} \quad \text { and } \quad y^{*}=J_{\lambda}^{B_{2}}\left(y^{*}\right),
$$

for some $\lambda>0$. Recently, SVIP (1.2)-(1.3) has received much attention and extensively investigated based on fixed-point algorithms due to its importance in convex optimization problems; see $[2,11,14,16]$ and the references therein.

Recently, Byrne et al. [2] studied the convergence of the following iterative method for solving SVIP (1.2)-(1.3):

$$
x_{1} \in H, \quad x_{n+1}=J_{\lambda}^{B_{1}}\left(x_{n}+\gamma A^{*}\left(J_{\lambda}^{B_{2}}-I\right) A x_{n}\right) \quad \forall n \geq 1, \exists \lambda>0 .
$$

Weak and strong convergence theorems were established in the framework of Hilbert spaces.

Let $T: C \rightarrow C$ be a nonlinear mapping. Denote by $\operatorname{Fix}(T)$ the fixed-point set of $T$, i.e., $\operatorname{Fix}(T)=$ $\{x \in C: x=T x\}$. Recall that $T$ is said to be $\kappa$-Lipschitzian if there exists a constant $\kappa>0$ such that $\|T x-T y\| \leq \kappa\|x-y\|, \forall x, y \in C$. In particular, if $\kappa=1$, then $T$ is said to be nonexpansive. If $\kappa<1$, then $T$ is said to be contractive.

For any $x \in H$, there exists a unique nearest point on the nonempty closed convex subset $C$ denoted by $P_{C} x$ such that $\left\|x-P_{C} x\right\| \leq\|x-y\|, \forall y \in C$. The mapping $P_{C}$ is called the metric projection of $H$ onto $C$. We know that $P_{C}$ is a nonexpansive mapping from $H$ onto $C$. The metric projection $P_{C}$ can be characterized by $P_{C} x \in C$ and

$$
\left\langle x-y, P_{C} x-P_{C} y\right\rangle \geq\left\|P_{C} x-P_{C} y\right\|^{2}, \quad \forall x, y \in H .
$$


For all $x \in H$ and $y \in C, P_{C} x$ is characterized by $\left\langle x-P_{C} x, y-P_{C} x\right\rangle \leq 0$. It is easy to see that the above inequality is equivalent to the following inequality:

$$
\|x-y\|^{2} \geq\left\|x-P_{C} x\right\|^{2}+\left\|y-P_{C} x\right\|^{2}, \quad \forall x \in H_{1}, y \in C .
$$

It is not hard to find that every nonexpansive mapping $T: H \rightarrow H$ satisfies the following inequality

$$
\langle(I-T) x-(I-T) y, T y-T x\rangle \leq \frac{1}{2}\|(I-T) x-(I-T) y\|^{2}, \quad \forall(x, y) \in H \times H .
$$

A mapping $T: H \rightarrow H$ is said to be averaged mapping if it can be written as an average of the identity $I$ and a nonexpansive mapping, that is,

$$
T=(1-\alpha) I+\alpha S,
$$

where $\alpha \in(0,1)$ and $S: H \rightarrow H$ is a nonexpansive mapping. We note that averaged mappings are nonexpansive. Further, firmly nonexpansive mappings (in particular, projections on nonempty closed and convex subsets and resolvent operators of maximal monotone operators).

The composite of finitely many averaged mappings is averaged. The following is a remarkable property of averaged mappings.

$$
\bigcap_{i=1}^{N} \operatorname{Fix}\left(T_{i}\right)=\operatorname{Fix}\left(T_{1} T_{2} \cdots T_{N-1} T_{N}\right)=\operatorname{Fix}\left(T_{2} T_{3} \cdots T_{N} T_{1}\right)=\cdots=\operatorname{Fix}\left(T_{N} T_{1} \cdots T_{N-2} T_{N-1}\right) .
$$

In particular, as $N=2$, we have $\operatorname{Fix}\left(T_{1} T_{2}\right)=\operatorname{Fix}\left(T_{2} T_{1}\right)=\operatorname{Fix}\left(T_{1}\right) \bigcap \operatorname{Fix}\left(T_{2}\right)$.

In 2014, Kazmi and Rizvi [14] studied SVIP (1.2)-(1.3) and fixed points of a nonexpansive mapping $S$ via the following iterative algorithm

$$
\left\{\begin{array}{l}
u_{n}=J_{\lambda}^{B_{1}}\left(x_{n}+\gamma A^{*}\left(J_{\lambda}^{B_{2}}-I\right) A x_{n}\right), \\
x_{n+1}=\alpha_{n} f\left(x_{n}\right)+\left(1-\alpha_{n}\right) S u_{n}, \quad \forall n \geq 0,
\end{array}\right.
$$

where $f$ is a contractive mapping in $H_{1}, \gamma \in\left(0, \frac{1}{L}\right)$ and $\left\{\alpha_{n}\right\}$ is a sequence in $(0,1)$ such that $\lim _{n \rightarrow \infty} \alpha_{n}=$ $0, \sum_{n=0}^{\infty} \alpha_{n}=\infty$ and $\sum_{n=1}^{\infty}\left|\alpha_{n}-\alpha_{n-1}\right|<\infty$. They proved that both $\left\{u_{n}\right\}$ and $\left\{x_{n}\right\}$ converge strongly to a point $z \in \operatorname{Sol}\left(B_{1}, B_{2}\right)$, which is the unique solution to the variational inequality $\langle(I-f) z, z-p\rangle \leq 0$, $\forall p \in \operatorname{Sol}\left(B_{1}, B_{2}\right)$.

Recall that a mapping $T$ is said to be pseudocontractive if

$$
\langle T x-T y, x-y\rangle \leq\|x-y\|^{2}, \quad \forall x, y \in C .
$$

$T$ is said to be strongly pseudocontractive if there exists a constant $\beta \in(0,1)$ such that

$$
\langle T x-T y, x-y\rangle \leq \beta\|x-y\|^{2}, \quad \forall x, y \in C .
$$

Let $\left\{S_{n}\right\}_{n=1}^{\infty}$ be a sequence of continuous pseudocontractive self mappings on $C$. Then $\left\{S_{n}\right\}_{n=1}^{\infty}$ is said to be a countable family of $\ell$-uniformly Lipschitzian pseudocontractive self-mappings on $C$ if there exists a constant $\ell>0$ such that each $S_{n}$ is $\ell$-Lipschitz continuous. Recall that a mapping $F: H \rightarrow H$ is said to be a strongly positive bounded linear operator if there exists a constant $\bar{\gamma}>0$ such that $\langle F x, x\rangle \geq \bar{\gamma}\|x\|^{2}$, $\forall x \in H$.

In this paper, we introduce a composite viscosity iterative algorithm for finding a common solution of a split variational inclusion problem and a fixed point of a family of pseudocontractive mappings. Strong convergence of the proposed iterative algorithm is obtained under some mild assumptions in the framework of Hilbert spaces. To prove our main results, we also need the following tools. 
Lemma 1.1 ([10]). Let $C$ be a nonempty closed convex subset of a Banach space $X$ and let $T: C \rightarrow C$ be a continuous and strong pseudocontraction mapping. Then, $T$ has a unique fixed point in $C$.

Lemma 1.2 ([22]). Let $H$ be a Hilbert space. Let $\lambda$ be a number in $(0,1]$ and let $T: H \rightarrow H$ be a nonexpansive mapping. Define a mapping $T^{\lambda}: H \rightarrow H$ by $T^{\lambda} x:=T x-\lambda \mu F(T x), \forall x \in H$, where $F: H \rightarrow H$ is a $\kappa$-Lipschitzian and $\eta$-strongly monotone operator. Then $T^{\lambda}$ is a contraction provided $0<\mu<\frac{2 \eta}{\kappa^{2}}$, that is, $\left\|T^{\lambda} x-T^{\lambda} y\right\| \leq(1-\lambda \tau)\|x-y\|, \forall x, y \in H$, where $\tau=1-\sqrt{1-\mu\left(2 \eta-\mu \kappa^{2}\right)} \in$ $(0,1]$.

Lemma 1.3 ([2]). Let $H_{1}$ and $H_{2}$ be two real Hilbert spaces. Let $B_{1}: H_{1} \rightarrow 2^{H_{1}}$ and $B_{2}: H_{2} \rightarrow 2^{H_{2}}$ be multi-valued maximal monotone mappings. Let $A$ be a bounded linear operator from $\mathrm{H}_{1}$ to $\mathrm{H}_{2}$ and let $A^{*}$ be its adjoint operator. Let $L$ be the spectral radius of the $A^{*} A$. For any given $\lambda>0$, let $G: H \rightarrow H$ be a mapping defined by $G:=J_{\lambda}^{B_{1}}\left(I+\gamma A^{*}\left(J_{\lambda}^{B_{2}}-I\right) A\right)$, where $\gamma \in\left(0, \frac{1}{L}\right)$. Then $G$ is a nonexpansive mapping. If $\operatorname{Sol}\left(B_{1}, B_{2}\right) \neq \emptyset$, then $\operatorname{Sol}\left(B_{1}, B_{2}\right)=\operatorname{Fix}(G)$.

Lemma 1.4 ([19]). Let $\left\{x_{n}\right\}$ and $\left\{z_{n}\right\}$ be bounded sequences in a Banach space $X$ and let $\left\{\beta_{n}\right\}$ be a sequence in $[0,1]$ with $0<\liminf _{n \rightarrow \infty} \beta_{n} \leq \limsup _{n \rightarrow \infty} \beta_{n}<1$. Suppose $x_{n+1}=\beta_{n} x_{n}+\left(1-\beta_{n}\right) z_{n}$ for all integers $n \geq 1$ and

$$
\limsup _{n \rightarrow \infty}\left(\left\|z_{n+1}-z_{n}\right\|-\left\|x_{n+1}-x_{n}\right\|\right) \leq 0
$$

Then, $\lim _{n \rightarrow \infty}\left\|z_{n}-x_{n}\right\|=0$.

Lemma 1.5 ([12]). Let $C$ be a nonempty closed convex subset of a Banach space X. Let $S_{1}, S_{2}, \ldots$ be a sequence of mappings of $C$ into itself. Suppose that $\sum_{n=1}^{\infty} \sup \left\{\left\|S_{n} x-S_{n+1} x\right\|: x \in C\right\}<\infty$. Then for each $y \in C,\left\{S_{n} y\right\}$ converges strongly to some point of $C$. Moreover, let $S$ be a mapping of $C$ into itself defined by $S y=\lim _{n \rightarrow \infty} S_{n} y$ for all $y \in C$. Then $\lim _{n \rightarrow \infty} \sup \left\{\left\|S x-S_{n} x\right\|: x \in C\right\}=0$.

Lemma 1.6 ([17]). Assume that $S$ is a nonexpansive self-mapping on a nonempty closed convex subset $C$ of a Hilbert space H. If $S$ has a fixed point, then I-S is demiclosed at zero, i.e., if $\left\{x_{n}\right\}$ is a sequence in $C$ converging weakly to some $x \in C$ and the sequence $\left\{(I-S) x_{n}\right\}$ converges strongly to zero, then $(I-S) x=0$, where $I$ is the identity mapping of $H_{1}$.

Lemma 1.7 ([23]). Let $\left\{a_{n}\right\}$ be a sequence of nonnegative numbers satisfying the conditions $a_{n+1} \leq$ $\left(1-\lambda_{n}\right) a_{n}+\lambda_{n} \gamma_{n}, \forall n \geq 1$, where $\left\{\lambda_{n}\right\}$ and $\left\{\gamma_{n}\right\}$ are sequences of real numbers such that $\left\{\lambda_{n}\right\} \subset[0,1]$ and $\sum_{n=1}^{\infty} \lambda_{n}=\infty, \limsup _{n \rightarrow \infty} \gamma_{n} \leq 0$. Then $\lim _{n \rightarrow \infty} a_{n}=0$.

Lemma 1.8 ([13]). Let $C$ be a nonempty closed convex subset of a real Hilbert space $H_{1}$, and let $B: C \rightarrow$ $H_{1}$ be a monotone and hemicontinuous mapping. Then the following hold:

(i) $\mathrm{VI}(C, B)=\left\{x^{*} \in C:\left\langle B y, y-x^{*}\right\rangle \geq 0, \forall y \in C\right\}$;

(ii) $\mathrm{VI}(C, B)=\operatorname{Fix}\left(P_{C}(I-\lambda B)\right)$ for all $\lambda>0$;

(iii) $\mathrm{VI}(C, B)$ consists of one point, if $B$ is strongly monotone and Lipschitz continuous.

\section{Main Results}

We are now in a position to state and prove the main result in this paper.

Theorem 2.1. Let $H_{1}$ and $H_{2}$ be real Hilbert spaces. Let $A: H_{1} \rightarrow H_{2}$ be a bounded linear operator and let $A^{*}$ be the adjoint of $A$. Let $L$ be the spectral radius of the operator $A^{*} A$. Suppose that $B_{1}: H_{1} \rightarrow 2^{H_{1}}$ 
and $B_{2}: H_{2} \rightarrow 2^{H_{2}}$ are maximal monotone mappings. Let $G: H_{1} \rightarrow H_{1}$ be a mapping defined as $G:=$ $J_{\lambda}^{B_{1}}\left(I+\gamma A^{*}\left(J_{\lambda}^{B_{2}}-I\right) A\right)$, where $\lambda>0, \gamma \in\left(0, \frac{1}{L}\right)$. Let $f: H_{1} \rightarrow H_{1}$ be a nonexpansive mapping and let $F: H_{1} \rightarrow H_{1}$ be $\kappa$-Lipschitzian and $\eta$-strongly monotone with constants $\kappa, \eta>0$ such that $0<\delta<\tau:=$ $1-\sqrt{1-\mu\left(2 \eta-\mu \kappa^{2}\right)} \in(0,1], 0<\mu<\frac{2 \eta}{\kappa^{2}}$. Assume that $\left\{S_{n}\right\}_{n=1}^{\infty}$ is a countable family of $\ell$-uniformly Lipschitzian pseudocontractive self-mappings on $H_{1}$ such that $\Omega:=\left(\bigcap_{n=1}^{\infty} \operatorname{Fix}\left(S_{n}\right)\right) \cap \operatorname{Sol}\left(B_{1}, B_{2}\right) \neq \emptyset$. For an arbitrary $x_{1} \in H_{1}$, let $\left\{x_{n}\right\}$ and $\left\{u_{n}\right\}$ be sequences generated by

$$
\left\{\begin{array}{l}
u_{n}=\gamma_{n} x_{n}+\left(1-\gamma_{n}\right) S_{n} u_{n}, \\
x_{n+1}=\alpha_{n} \delta f\left(x_{n}\right)+\beta_{n} x_{n}+\left[\left(1-\beta_{n}\right) I-\alpha_{n} \mu F\right] G u_{n}, \quad \forall n \geq 1,
\end{array}\right.
$$

where $\left\{\alpha_{n}\right\},\left\{\beta_{n}\right\}$ and $\left\{\gamma_{n}\right\}$ are the sequences in $(0,1)$ satisfying the following conditions:

(1) $\left\{\alpha_{n}+\beta_{n}\right\} \subset(0,1]$ and $\left\{\beta_{n}\right\}_{n=1}^{\infty} \subset[a, b]$ for some $a, b \in(0,1)$;

(2) $\lim _{n \rightarrow \infty} \alpha_{n}=0$ and $\sum_{n=1}^{\infty} \alpha_{n}=\infty$;

(3) $0<\liminf _{n \rightarrow \infty} \gamma_{n} \leq \limsup _{n \rightarrow \infty} \gamma_{n}<1$ and $\lim _{n \rightarrow \infty}\left|\gamma_{n+1}-\gamma_{n}\right|=0$.

Assume that $\sum_{n=1}^{\infty} \sup _{x \in K}\left\|S_{n} x-S_{n+1} x\right\|<\infty$ for any bounded subset $K$ of $H_{1}$. Let $S$ be a mapping of $H_{1}$ into itself defined by $S x=\lim _{n \rightarrow \infty} S_{n} x$ for all $x \in H_{1}$ with $\operatorname{Fix}(S)=\bigcap_{n=1}^{\infty} \operatorname{Fix}\left(S_{n}\right)$. Then $\left\{x_{n}\right\}$ and $\left\{u_{n}\right\}$ converge strongly to a point $z \in \Omega$, which is the unique solution to the variational inequality

$$
\langle(\mu F-\delta f) z, z-p\rangle \leq 0, \quad \forall p \in \Omega .
$$

Proof. Taking into account $0<\liminf _{n \rightarrow \infty} \gamma_{n} \leq \limsup _{n \rightarrow \infty} \gamma_{n}<1$, we may assume, without loss of generality, that $\left\{\gamma_{n}\right\} \subset[c, d] \subset(0,1)$ for some $c, d \in(0,1)$. Define a mapping $F_{n}$ by $F_{n} x=\gamma_{n} x_{n}+\left(1-\gamma_{n}\right) S_{n} x$, $\forall x \in H_{1}$. Since each $S_{n}: H_{1} \rightarrow H_{1}$ is a continuous pseudocontraction mapping, we deduce that

$$
\left\langle F_{n} x-F_{n} y, x-y\right\rangle \leq\left(1-\gamma_{n}\right)\|x-y\|^{2}, \quad \forall x, y \in H_{1} .
$$

This shows that $F_{n}$ is a continuous strong pseudocontraction. From Lemma 1.1, we know that, for each $n \geq 1, u_{n}=\gamma_{n} x_{n}+\left(1-\gamma_{n}\right) S_{n} u_{n}$ in (2.1) is well defined.

We now claim that $\left\{x_{n}\right\},\left\{y_{n}\right\},\left\{u_{n}\right\},\left\{S_{n} u_{n}\right\},\left\{f\left(x_{n}\right)\right\}$ and $\left\{F\left(y_{n}\right)\right\}$ are bounded. Indeed, take an element $p \in \Omega$ arbitrarily. Then $p=J_{\lambda}^{B_{1}} p, A p=J_{\lambda}^{B_{2}}(A p)$ and $S_{n} p=p$ for all $n \geq 1$. Since each $S_{n}: H_{1} \rightarrow$ $H_{1}$ is a pseudocontraction mapping, it follows that

$$
\begin{aligned}
\left\|u_{n}-p\right\|^{2} & =\left\langle u_{n}-p, u_{n}-p\right\rangle \\
& =\gamma_{n}\left\langle x_{n}-p, u_{n}-p\right\rangle+\left(1-\gamma_{n}\right)\left\langle S_{n} u_{n}-p, u_{n}-p\right\rangle \\
& \leq \gamma_{n}\left\|x_{n}-p\right\|\left\|u_{n}-p\right\|+\left(1-\gamma_{n}\right)\left\|u_{n}-p\right\|^{2},
\end{aligned}
$$

which yields $\left\|u_{n}-p\right\| \leq\left\|x_{n}-p\right\|$. Putting

$$
y_{n}=J_{\lambda}^{B_{1}}\left(u_{n}+\gamma A^{*}\left(J_{\lambda}^{B_{2}}-I\right) A u_{n}\right),
$$

we obtain

$$
\begin{aligned}
\left\|y_{n}-p\right\|^{2} & =\left\|J_{\lambda}^{B_{1}}\left(u_{n}+\gamma A^{*}\left(J_{\lambda}^{B_{2}}-I\right) A u_{n}\right)-J_{\lambda}^{B_{1}} p\right\|^{2} \\
& \leq\left\|u_{n}+\gamma A^{*}\left(J_{\lambda}^{B_{2}}-I\right) A u_{n}-p\right\|^{2} \\
& =\left\|u_{n}-p\right\|^{2}+\gamma^{2}\left\|A^{*}\left(J_{\lambda}^{B_{2}}-I\right) A u_{n}\right\|^{2}+2 \gamma\left\langle u_{n}-p, A^{*}\left(J_{\lambda}^{B_{2}}-I\right) A u_{n}\right\rangle .
\end{aligned}
$$

Thus,

$$
\left\|y_{n}-p\right\|^{2} \leq\left\|x_{n}-p\right\|^{2}+\gamma^{2}\left\langle\left(J_{\lambda}^{B_{2}}-I\right) A u_{n}, A A^{*}\left(J_{\lambda}^{B_{2}}-I\right) A u_{n}\right\rangle+2 \gamma\left\langle u_{n}-p, A^{*}\left(J_{\lambda}^{B_{2}}-I\right) A u_{n}\right\rangle .
$$


Note that

$$
\gamma^{2}\left\langle\left(J_{\lambda}^{B_{2}}-I\right) A u_{n}, A A^{*}\left(J_{\lambda}^{B_{2}}-I\right) A u_{n}\right\rangle \leq L \gamma^{2}\left\|\left(J_{\lambda}^{B_{2}}-I\right) A u_{n}\right\|^{2} .
$$

From (1.4), we have

$$
\begin{aligned}
& 2 \gamma\left\langle u_{n}-p, A^{*}\left(J_{\lambda}^{B_{2}}-I\right) A u_{n}\right\rangle \\
& =2 \gamma\left\langle A\left(u_{n}-p\right)+\left(J_{\lambda}^{B_{2}}-I\right) A u_{n}-\left(J_{\lambda}^{B_{2}}-I\right) A u_{n},\left(J_{\lambda}^{B_{2}}-I\right) A u_{n}\right\rangle \\
& =2 \gamma\left\{\left\langle A p-J_{\lambda}^{B_{2}} A u_{n}, A u_{n}-J_{\lambda}^{B_{2}} A u_{n}\right\rangle-\left\|\left(J_{\lambda}^{B_{2}}-I\right) A u_{n}\right\|^{2}\right\} \\
& \leq 2 \gamma\left\{\frac{1}{2}\left\|\left(J_{\lambda}^{B_{2}}-I\right) A u_{n}\right\|^{2}-\left\|\left(J_{\lambda}^{B_{2}}-I\right) A u_{n}\right\|^{2}\right\} \\
& =-\gamma\left\|\left(J_{\lambda}^{B_{2}}-I\right) A u_{n}\right\|^{2} .
\end{aligned}
$$

It follows that

$$
\left\|y_{n}-p\right\|^{2} \leq\left\|x_{n}-p\right\|^{2}+\gamma(L \gamma-1)\left\|\left(J_{\lambda}^{B_{2}}-I\right) A u_{n}\right\|^{2} .
$$

From $\gamma \in\left(0, \frac{1}{L}\right)$, we arrive at $\left\|y_{n}-p\right\| \leq\left\|x_{n}-p\right\|$. Since $f: H_{1} \rightarrow H_{1}$ is nonexpansive, we conclude that

$$
\begin{aligned}
\left\|x_{n+1}-p\right\| \leq & \left\|\alpha_{n}\left(\delta f\left(x_{n}\right)-\mu F p\right)+\beta_{n}\left(x_{n}-p\right)+\left[\left(1-\beta_{n}\right) I-\alpha_{n} \mu F\right] y_{n}-\left[\left(1-\beta_{n}\right) I-\alpha_{n} \mu F\right] p\right\| \\
\leq & \alpha_{n} \delta\left\|f\left(x_{n}\right)-f(p)\right\|+\alpha_{n}\|\delta f(p)-\mu F p\|+\beta_{n}\left\|x_{n}-p\right\| \\
& +\left(1-\beta_{n}\right)\left\|\left[I-\frac{\alpha_{n}}{1-\beta_{n}} \mu F\right] y_{n}-\left[I-\frac{\alpha_{n}}{1-\beta_{n}} \mu F\right] p\right\| \\
\leq & \alpha_{n} \delta\left\|x_{n}-p\right\|+\alpha_{n}\|\delta f(p)-\mu F p\|+\beta_{n}\left\|x_{n}-p\right\|+\left(1-\beta_{n}\right)\left(1-\frac{\alpha_{n}}{1-\beta_{n}} \tau\right)\left\|y_{n}-p\right\| \\
\leq & \alpha_{n} \delta\left\|x_{n}-p\right\|+\alpha_{n}\|\delta f(p)-\mu F p\|+\beta_{n}\left\|x_{n}-p\right\|+\left(1-\beta_{n}-\alpha_{n} \tau\right)\left\|x_{n}-p\right\| \\
= & {\left[1-\alpha_{n}(\tau-\delta)\right]\left\|x_{n}-p\right\|+\alpha_{n}(\tau-\delta) \cdot \frac{\|\delta f(p)-\mu F p\|}{\tau-\delta} } \\
\leq & \max \left\{\left\|x_{n}-p\right\|, \frac{\|\delta f(p)-\mu F p\|}{\tau-\delta}\right\} .
\end{aligned}
$$

By induction, we have

$$
\left\|x_{n}-p\right\| \leq \max \left\{\left\|x_{1}-p\right\|, \frac{\|\delta f(p)-\mu F p\|}{\tau-\delta}\right\}, \quad \forall n \geq 1 .
$$

It immediately follows that $\left\{x_{n}\right\}$ is bounded, so are $\left\{y_{n}\right\},\left\{u_{n}\right\},\left\{f\left(x_{n}\right)\right\}$ and $\left\{F\left(y_{n}\right)\right\}$. Taking into account that $\left\{S_{n}\right\}$ is $\ell$-uniformly Lipschitzian on $C$, we know that

$$
\left\|S_{n} u_{n}\right\| \leq\left\|S_{n} u_{n}-p\right\|+\|p\| \leq \ell\left\|u_{n}-p\right\|+\|p\|,
$$

which implies that $\left\{S_{n} u_{n}\right\}$ is also bounded. Setting $x_{n+1}=\beta_{n} x_{n}+\left(1-\beta_{n}\right) v_{n}$, we see that

$$
\begin{aligned}
v_{n} & =\frac{1}{1-\beta_{n}}\left\{\alpha_{n} \delta f\left(x_{n}\right)+\beta_{n} x_{n}+\left[\left(1-\beta_{n}\right) I-\alpha_{n} \mu F\right] y_{n}\right\}-\frac{\beta_{n}}{1-\beta_{n}} x_{n} \\
& =\frac{1}{1-\beta_{n}}\left\{\alpha_{n} \delta f\left(x_{n}\right)+\beta_{n} x_{n}+y_{n}-\beta_{n} y_{n}-\alpha_{n} \mu F y_{n}\right\}-\frac{\beta_{n}}{1-\beta_{n}} x_{n} \\
& =\frac{1}{1-\beta_{n}}\left\{\alpha_{n}\left(\delta f\left(x_{n}\right)-\mu F y_{n}\right)+\left(1-\beta_{n}\right) y_{n}\right\} \\
& =\frac{\alpha_{n}}{1-\beta_{n}}\left(\delta f\left(x_{n}\right)-\mu F y_{n}\right)+y_{n} .
\end{aligned}
$$

Hence,

$$
\left\|v_{n+1}-v_{n}\right\| \leq\left\|y_{n+1}-y_{n}\right\|+\frac{\alpha_{n+1}}{1-\beta_{n+1}}\left\|\delta f\left(x_{n+1}\right)-\mu F y_{n+1}\right\|+\frac{\alpha_{n}}{1-\beta_{n}}\left\|\delta f\left(x_{n}\right)-\mu F y_{n}\right\| .
$$

By using Lemma 1.3, we know that $G:=J_{\lambda}^{B_{1}}\left(I+\gamma A^{*}\left(J_{\lambda}^{B_{2}}-I\right) A\right)$ is nonexpansive. So

$$
\left\|y_{n+1}-y_{n}\right\|=\left\|G u_{n+1}-G u_{n}\right\| \leq\left\|u_{n+1}-u_{n}\right\| .
$$


On the other hand, simple calculations show that

$$
\begin{aligned}
\left\|u_{n+1}-u_{n}\right\|^{2} & =\gamma_{n+1}\left\langle x_{n+1}-x_{n}, u_{n+1}-u_{n}\right\rangle+\left(1-\gamma_{n+1}\right)\left[\left\langle S_{n+1} u_{n+1}-S_{n} u_{n+1}, u_{n+1}-u_{n}\right\rangle\right. \\
& \left.+\left\langle S_{n} u_{n+1}-S_{n} u_{n}, u_{n+1}-u_{n}\right\rangle\right]+\left(\gamma_{n+1}-\gamma_{n}\right)\left\langle x_{n}-S_{n} u_{n}, u_{n+1}-u_{n}\right\rangle \\
& \leq \gamma_{n+1}\left\|x_{n+1}-x_{n}\right\|\left\|u_{n+1}-u_{n}\right\|+\left(1-\gamma_{n+1}\right)\left[\left\|S_{n+1} u_{n+1}-S_{n} u_{n+1}\right\|\left\|u_{n+1}-u_{n}\right\|\right. \\
& \left.+\left\|u_{n+1}-u_{n}\right\|^{2}\right]+\left|\gamma_{n+1}-\gamma_{n}\right|\left\|x_{n}-S_{n} u_{n}\right\|\left\|u_{n+1}-u_{n}\right\|,
\end{aligned}
$$

that is,

$$
\begin{aligned}
\left\|u_{n+1}-u_{n}\right\| & \leq \gamma_{n+1}\left\|x_{n+1}-x_{n}\right\|+\left(1-\gamma_{n+1}\right)\left[\left\|S_{n+1} u_{n+1}-S_{n} u_{n+1}\right\|\right. \\
& \left.+\left\|u_{n+1}-u_{n}\right\|\right]+\left|\gamma_{n+1}-\gamma_{n}\right|\left\|x_{n}-S_{n} u_{n}\right\|,
\end{aligned}
$$

which immediately leads to

$$
\begin{aligned}
\left\|u_{n+1}-u_{n}\right\| & \leq\left\|x_{n+1}-x_{n}\right\|+\frac{1-\gamma_{n+1}}{\gamma_{n+1}}\left\|S_{n+1} u_{n+1}-S_{n} u_{n+1}\right\|+\left|\gamma_{n+1}-\gamma_{n}\right| \frac{\left\|x_{n}-S_{n} u_{n}\right\|}{\gamma_{n+1}} \\
& \leq\left\|x_{n+1}-x_{n}\right\|+\frac{1}{c}\left\|S_{n+1} u_{n+1}-S_{n} u_{n+1}\right\|+\left|\gamma_{n+1}-\gamma_{n}\right| \frac{\left\|x_{n}-S_{n} u_{n}\right\|}{c} .
\end{aligned}
$$

Putting $K:=\left\{u_{n}: n \geq 1\right\}$, we know that $K$ is a bounded subset of $H_{1}$. By the assumption, we get $\sum_{n=1}^{\infty} \sup _{x \in K}\left\|S_{n+1} x-S_{n} x\right\|<\infty$. In view of

$$
\left\|S_{n+1} u_{n+1}-S_{n} u_{n+1}\right\| \leq \sup _{x \in K}\left\|S_{n+1} x-S_{n} x\right\|,
$$

we have

$$
\sum_{n=1}^{\infty}\left\|S_{n+1} u_{n+1}-S_{n} u_{n+1}\right\|<\infty
$$

Therefore,

$$
\begin{aligned}
\left\|v_{n+1}-v_{n}\right\| & \leq \frac{\alpha_{n+1}}{1-\beta_{n+1}}\left\|\delta f\left(x_{n+1}\right)-\mu F y_{n+1}\right\|+\frac{\alpha_{n}}{1-\beta_{n}}\left\|\delta f\left(x_{n}\right)-\mu F y_{n}\right\|+\left\|y_{n+1}-y_{n}\right\| \\
& \leq \frac{\alpha_{n+1}}{1-\beta_{n+1}}\left\|\delta f\left(x_{n+1}\right)-\mu F y_{n+1}\right\|+\frac{\alpha_{n}}{1-\beta_{n}}\left\|\delta f\left(x_{n}\right)-\mu F y_{n}\right\|+\left\|u_{n+1}-u_{n}\right\| \\
& \leq \frac{\alpha_{n+1}}{1-\beta_{n+1}}\left\|\delta f\left(x_{n+1}\right)-\mu F y_{n+1}\right\|+\frac{\alpha_{n}}{1-\beta_{n}}\left\|\delta f\left(x_{n}\right)-\mu F y_{n}\right\|+\left\|x_{n+1}-x_{n}\right\| \\
& +\frac{1}{c}\left\|S_{n+1} u_{n+1}-S_{n} u_{n+1}\right\|+\left|\gamma_{n+1}-\gamma_{n}\right| \frac{\left\|x_{n}-S_{n} u_{n}\right\|}{c},
\end{aligned}
$$

which immediately yields

$$
\begin{aligned}
\left\|v_{n+1}-v_{n}\right\|-\left\|x_{n+1}-x_{n}\right\| & \leq \frac{\alpha_{n+1}}{1-\beta_{n+1}}\left\|\delta f\left(x_{n+1}\right)-\mu F y_{n+1}\right\|+\frac{\alpha_{n}}{1-\beta_{n}}\left\|\delta f\left(x_{n}\right)-\mu F y_{n}\right\| \\
& +\frac{1}{c}\left\|S_{n+1} u_{n+1}-S_{n} u_{n+1}\right\|+\left|\gamma_{n+1}-\gamma_{n}\right| \frac{\left\|x_{n}-S_{n} u_{n}\right\|}{c} .
\end{aligned}
$$

It follows that $\limsup _{n \rightarrow \infty}\left(\left\|v_{n+1}-v_{n}\right\|-\left\|x_{n+1}-x_{n}\right\|\right) \leq 0$. An application of Lemma 1.4 yields that

$$
\lim _{n \rightarrow \infty}\left\|v_{n}-x_{n}\right\|=0
$$

Since

$$
\left\|x_{n+1}-x_{n}\right\|=\left\|\gamma_{n} x_{n}+\left(1-\gamma_{n}\right) v_{n}-\left[\gamma_{n} x_{n}+\left(1-\gamma_{n}\right) x_{n}\right]\right\|=\left(1-\gamma_{n}\right)\left\|v_{n}-x_{n}\right\|,
$$

we conclude from (2.4) that

$$
\lim _{n \rightarrow \infty}\left\|x_{n+1}-x_{n}\right\|=0
$$

so are

$$
\lim _{n \rightarrow \infty}\left\|u_{n+1}-u_{n}\right\|=0 \quad \text { and } \quad \lim _{n \rightarrow \infty}\left\|y_{n+1}-y_{n}\right\|=0
$$


Set $f_{n}=\delta f\left(x_{n}\right)-\mu F y_{n}$ for all $n \geq 1$. For any $p \in \Omega$, we observe that

$$
\begin{aligned}
\left\|x_{n+1}-p\right\|^{2} & =\left\|\alpha_{n} \delta f\left(x_{n}\right)+\beta_{n} x_{n}+\left(1-\beta_{n}\right) y_{n}-\alpha_{n} \mu F y_{n}-p\right\|^{2} \\
& =\left\|\alpha_{n} f_{n}+\beta_{n} x_{n}+\left(1-\beta_{n}\right) y_{n}-\beta_{n} p-\left(1-\beta_{n}\right) p\right\|^{2} \\
& \leq\left\|\beta_{n}\left(x_{n}-p\right)+\left(1-\beta_{n}\right)\left(y_{n}-p\right)\right\|^{2}+2\left\langle\alpha_{n} f_{n}, x_{n+1}-p\right\rangle \\
& \leq \beta_{n}\left\|x_{n}-p\right\|^{2}+\left(1-\beta_{n}\right)\left\|y_{n}-p\right\|^{2}-\beta_{n}\left(1-\beta_{n}\right)\left\|x_{n}-y_{n}\right\|^{2} \\
& +2 \alpha_{n}\left\|f_{n}\right\|\left\|x_{n+1}-p\right\| \\
& \leq \beta_{n}\left\|x_{n}-p\right\|^{2}+\left(1-\beta_{n}\right)\left\|y_{n}-p\right\|^{2}+2 \alpha_{n} M^{2},
\end{aligned}
$$

where $M=\max \left\{\sup _{n>1}\left\|f_{n}\right\|, \sup _{n \geq 1}\left\|x_{n}-p\right\|\right\}$. Substituting (2.3) into (2.7), we obtain that

$$
\begin{aligned}
\left\|x_{n+1}-p\right\|^{2} & \leq \beta_{n}\left\|x_{n}-p\right\|^{2}+\left(1-\beta_{n}\right)\left[\left\|u_{n}-p\right\|^{2}+\gamma(L \gamma-1)\left\|\left(J_{\lambda}^{B_{2}}-I\right) A u_{n}\right\|^{2}\right]+2 \alpha_{n} M^{2} \\
& \leq\left\|x_{n}-p\right\|^{2}-\gamma\left(1-\beta_{n}\right)(1-L \gamma)\left\|\left(J_{\lambda}^{B_{2}}-I\right) A u_{n}\right\|^{2}+2 \alpha_{n} M^{2} .
\end{aligned}
$$

Therefore,

$$
\begin{aligned}
\gamma\left(1-\beta_{n}\right)(1-L \gamma)\left\|\left(J_{\lambda}^{B_{2}}-I\right) A u_{n}\right\|^{2} & \leq\left\|x_{n}-p\right\|^{2}-\left\|x_{n+1}-p\right\|^{2}+2 \alpha_{n} M^{2} \\
& \leq\left(\left\|x_{n}-p\right\|+\left\|x_{n+1}-p\right\|\right)\left\|x_{n}-x_{n+1}\right\|+2 \alpha_{n} M^{2},
\end{aligned}
$$

From conditions (1), (2), and (2.5), we get

$$
\lim _{n \rightarrow \infty}\left\|\left(J_{\lambda}^{B_{2}}-I\right) A u_{n}\right\|=0
$$

Since $J_{\lambda}^{B_{1}}$ is firmly nonexpansive mapping, we conclude from inequality (2.2) that

$$
\begin{aligned}
\left\|y_{n}-p\right\|^{2} & \leq\left\langle y_{n}-p, u_{n}+\gamma A^{*}\left(J_{\lambda}^{B_{2}}-I\right) A u_{n}-p\right\rangle \\
& =\frac{1}{2}\left\{\left\|y_{n}-p\right\|^{2}+\left\|u_{n}+\gamma A^{*}\left(J_{\lambda}^{B_{2}}-I\right) A u_{n}-p\right\|^{2}-\| y_{n}-p\right. \\
& \left.-\left[u_{n}+\gamma A^{*}\left(J_{\lambda}^{B_{2}}-I\right) A u_{n}-p\right] \|^{2}\right\} \\
& \leq \frac{1}{2}\left\{\left\|y_{n}-p\right\|^{2}+\left\|u_{n}-p\right\|^{2}-\gamma\left\|\left(J_{\lambda}^{B_{2}}-I\right) A u_{n}\right\|^{2}+\gamma^{2}\left\|A^{*}\left(J_{\lambda}^{B_{2}}-I\right) A u_{n}\right\|^{2}\right. \\
& \left.-\left\|y_{n}-u_{n}-\gamma A^{*}\left(J_{\lambda}^{B_{2}}-I\right) A u_{n}\right\|^{2}\right\} \\
& =\frac{1}{2}\left\{\left\|y_{n}-p\right\|^{2}+\left\|u_{n}-p\right\|^{2}-\gamma\left\|\left(J_{\lambda}^{B_{2}}-I\right) A u_{n}\right\|^{2}+\gamma^{2}\left\|A^{*}\left(J_{\lambda}^{B_{2}}-I\right) A u_{n}\right\|^{2}\right. \\
& \left.-\left\|y_{n}-u_{n}\right\|^{2}-\gamma^{2}\left\|A^{*}\left(J_{\lambda}^{B_{2}}-I\right) A u_{n}\right\|^{2}+2 \gamma\left\langle y_{n}-u_{n}, A^{*}\left(J_{\lambda}^{B_{2}}-I\right) A u_{n}\right\rangle\right\} \\
& \leq \frac{1}{2}\left\{\left\|y_{n}-p\right\|^{2}+\left\|u_{n}-p\right\|^{2}-\left\|y_{n}-u_{n}\right\|^{2}+2 \gamma\left\langle y_{n}-u_{n}, A^{*}\left(J_{\lambda}^{B_{2}}-I\right) A u_{n}\right\rangle\right\} \\
& \leq \frac{1}{2}\left\{\left\|y_{n}-p\right\|^{2}+\left\|u_{n}-p\right\|^{2}-\left\|y_{n}-u_{n}\right\|^{2}+2 \gamma\left\|A\left(y_{n}-u_{n}\right)\right\|\left\|\left(J_{\lambda}^{B_{2}}-I\right) A u_{n}\right\|\right\} .
\end{aligned}
$$

Hence, we obtain

$$
\left\|y_{n}-p\right\|^{2} \leq\left\|u_{n}-p\right\|^{2}-\left\|y_{n}-u_{n}\right\|^{2}+2 \gamma\left\|A\left(y_{n}-u_{n}\right)\right\|\left\|\left(J_{\lambda}^{B_{2}}-I\right) A u_{n}\right\| .
$$

Substituting (2.9) into (2.7), one concludes that

$$
\begin{aligned}
\left\|x_{n+1}-p\right\|^{2} & \leq \beta_{n}\left\|x_{n}-p\right\|^{2}+\left(1-\beta_{n}\right)\left[\left\|u_{n}-p\right\|^{2}-\left\|y_{n}-u_{n}\right\|^{2}\right. \\
& \left.+2 \gamma\left\|A\left(y_{n}-u_{n}\right)\right\|\left\|\left(J_{\lambda}^{B_{2}}-I\right) A u_{n}\right\|\right]+2 \alpha_{n} M^{2} \\
& \leq\left\|x_{n}-p\right\|^{2}-\left(1-\beta_{n}\right)\left\|y_{n}-u_{n}\right\|^{2} \\
& +2 \gamma\left(1-\beta_{n}\right)\left\|A\left(y_{n}-u_{n}\right)\right\|\left\|\left(J_{\lambda}^{B_{2}}-I\right) A u_{n}\right\|+2 \alpha_{n} M^{2} .
\end{aligned}
$$

So,

$$
\begin{aligned}
\left(1-\beta_{n}\right)\left\|y_{n}-u_{n}\right\|^{2} & \leq\left(\left\|x_{n}-p\right\|+\left\|x_{n+1}-p\right\|\right)\left\|x_{n}-x_{n+1}\right\| \\
& +2 \gamma\left(1-\beta_{n}\right)\left\|A\left(y_{n}-u_{n}\right)\right\|\left\|\left(J_{\lambda}^{B_{2}}-I\right) A u_{n}\right\|+2 \alpha_{n} M^{2} .
\end{aligned}
$$


From conditions (1), (2), (2.5), and (2.8), we obtain that $\lim _{n \rightarrow \infty}\left\|y_{n}-u_{n}\right\|=0$. Since each $S_{n}$ is a pseudocontractive mapping, we have

$$
\begin{aligned}
\left\|u_{n}-p\right\|^{2} & =\left\langle\gamma_{n}\left(x_{n}-p\right)+\left(1-\gamma_{n}\right)\left(S_{n} u_{n}-p\right), u_{n}-p\right\rangle \\
& =\gamma_{n}\left\langle x_{n}-p, u_{n}-p\right\rangle+\left(1-\gamma_{n}\right)\left\langle S_{n} u_{n}-p, u_{n}-p\right\rangle \\
& \leq \gamma_{n}\left\langle x_{n}-p, u_{n}-p\right\rangle+\left(1-\gamma_{n}\right)\left\|u_{n}-p\right\|^{2},
\end{aligned}
$$

which immediately leads to

$$
\begin{aligned}
\left\|u_{n}-p\right\|^{2} & \leq\left\langle x_{n}-p, u_{n}-p\right\rangle \\
& =\frac{1}{2}\left[\left\|x_{n}-p\right\|^{2}+\left\|u_{n}-p\right\|^{2}-\left\|x_{n}-u_{n}\right\|^{2}\right] .
\end{aligned}
$$

Hence,

$$
\left\|y_{n}-p\right\|^{2} \leq\left\|u_{n}-p\right\|^{2} \leq\left\|x_{n}-p\right\|^{2}-\left\|x_{n}-u_{n}\right\|^{2},
$$

which, together with (2.7), yields

$$
\begin{aligned}
\left\|x_{n+1}-p\right\|^{2} & \leq \beta_{n}\left\|x_{n}-p\right\|^{2}+\left(1-\beta_{n}\right)\left\|y_{n}-p\right\|^{2}-\beta_{n}\left(1-\beta_{n}\right)\left\|x_{n}-y_{n}\right\|^{2}+2 \alpha_{n} M^{2} \\
& \leq \beta_{n}\left\|x_{n}-p\right\|^{2}+\left(1-\beta_{n}\right)\left[\left\|x_{n}-p\right\|^{2}-\left\|x_{n}-u_{n}\right\|^{2}\right]-\beta_{n}\left(1-\beta_{n}\right)\left\|x_{n}-y_{n}\right\|^{2}+2 \alpha_{n} M^{2} \\
& =\left\|x_{n}-p\right\|^{2}-\left(1-\beta_{n}\right)\left\|x_{n}-u_{n}\right\|^{2}-\beta_{n}\left(1-\beta_{n}\right)\left\|x_{n}-y_{n}\right\|^{2}+2 \alpha_{n} M^{2} .
\end{aligned}
$$

This implies that

$$
\begin{aligned}
& \left(1-\beta_{n}\right)\left\|x_{n}-u_{n}\right\|^{2}+\beta_{n}\left(1-\beta_{n}\right)\left\|x_{n}-y_{n}\right\|^{2} \\
& \leq\left\|x_{n}-p\right\|^{2}-\left\|x_{n+1}-p\right\|^{2}+2 \alpha_{n} M^{2} \\
& \leq\left(\left\|x_{n}-p\right\|+\left\|x_{n+1}-p\right\|\right)\left\|x_{n}-x_{n+1}\right\|+2 \alpha_{n} M^{2} .
\end{aligned}
$$

From conditions (1), (2), and (2.5), we get

$$
\lim _{n \rightarrow \infty}\left\|x_{n}-u_{n}\right\|=0 \quad \text { and } \quad \lim _{n \rightarrow \infty}\left\|x_{n}-y_{n}\right\|=0 .
$$

Noticing that $\left\|u_{n}-x_{n}\right\|=\left(1-\gamma_{n}\right)\left\|S_{n} u_{n}-x_{n}\right\| \geq(1-d)\left\|S_{n} u_{n}-x_{n}\right\|$,

$$
\left\|x_{n}-S_{n} x_{n}\right\| \leq\left\|x_{n}-S_{n} u_{n}\right\|+\left\|S_{n} u_{n}-S_{n} x_{n}\right\| \leq\left\|x_{n}-S_{n} u_{n}\right\|+\ell\left\|u_{n}-x_{n}\right\|,
$$

and

$$
\left\|x_{n}-G x_{n}\right\| \leq\left\|x_{n}-u_{n}\right\|+\left\|u_{n}-y_{n}\right\|+\left\|G u_{n}-G x_{n}\right\| \leq 2\left\|x_{n}-u_{n}\right\|+\left\|u_{n}-y_{n}\right\|,
$$

we deduce from (2.10) that

$$
\lim _{n \rightarrow \infty}\left\|x_{n}-S_{n} u_{n}\right\|=0, \quad \lim _{n \rightarrow \infty}\left\|x_{n}-S_{n} x_{n}\right\|=0 \quad \text { and } \quad \lim _{n \rightarrow \infty}\left\|x_{n}-G x_{n}\right\|=0 .
$$

Next, we claim that $\left\|x_{n}-\bar{S} x_{n}\right\| \rightarrow 0$ as $n \rightarrow \infty$, where $\bar{S}:=(2 I-S)^{-1}$. First, let us show that $S: H_{1} \rightarrow$ $H_{1}$ is pseudocontractive and $\ell$-Lipschitzian such that $\lim _{n \rightarrow \infty}\left\|S x_{n}-x_{n}\right\|=0$, where $S x=\lim _{n \rightarrow \infty} S_{n} x$, $\forall x \in H_{1}$. Observe that, for all $x, y \in H_{1}, \lim _{n \rightarrow \infty}\left\|S_{n} x-S x\right\|=0$ and $\lim _{n \rightarrow \infty}\left\|S_{n} y-S y\right\|=0$. Since each $S_{n}$ is pseudocontractive, we get

$$
\langle S x-S y, x-y\rangle=\lim _{n \rightarrow \infty}\left\langle S_{n} x-S_{n} y, x-y\right\rangle \leq\|x-y\|^{2} .
$$

This means that $S$ is pseudocontractive. Because $\left\{S_{n}\right\}_{n=1}^{\infty}$ is $\ell$-uniformly Lipschitzian on $H_{1}$, we have

$$
\|S x-S y\|=\lim _{n \rightarrow \infty}\left\|S_{n} x-S_{n} y\right\| \leq \ell\|x-y\|, \quad \forall x, y \in H_{1} .
$$


This means that $S$ is $\ell$-Lipschitzian. Taking into account the boundedness of $\left\{x_{n}\right\}$ and putting $K=$ $\overline{\operatorname{conv}}\left\{x_{n}: n \geq 1\right\}$ (the closed convex hull of the set $\left\{x_{n}: n \geq 1\right\}$ ), we have $\sum_{n=1}^{\infty} \sup _{x \in K}\left\|S_{n} x-S_{n+1} x\right\|<\infty$. Hence, by Lemma 1.5, we get $\lim _{n \rightarrow \infty} \sup _{x \in K}\left\|S_{n} x-S x\right\|=0$, which immediately yields

$$
\lim _{n \rightarrow \infty}\left\|S_{n} x_{n}-S x_{n}\right\|=0 .
$$

Combining (2.11) with (2.12) we have $\left\|x_{n}-S x_{n}\right\| \leq\left\|x_{n}-S_{n} x_{n}\right\|+\left\|S_{n} x_{n}-S x_{n}\right\| \rightarrow 0$ as $n \rightarrow \infty$, that is,

$$
\lim _{n \rightarrow \infty}\left\|x_{n}-S x_{n}\right\|=0
$$

Define $\bar{S}:=(2 I-S)^{-1}$. Then $\bar{S}: H_{1} \rightarrow H_{1}$ is nonexpansive with $\operatorname{Fix}(\bar{S})=\operatorname{Fix}(S)=\bigcap_{n=1}^{\infty} \operatorname{Fix}\left(S_{n}\right)$ and $\lim _{n \rightarrow \infty}\left\|x_{n}-\bar{S} x_{n}\right\|=0$. Indeed, put $\bar{S}:=(2 I-S)^{-1}$, where $I$ is the identity mapping of $H_{1}$. Then it is known that $\bar{S}$ is nonexpansive and $\operatorname{Fix}(\bar{S})=\operatorname{Fix}(S)=\bigcap_{n=1}^{\infty} \operatorname{Fix}\left(S_{n}\right)$ as a consequence of [15, Theorem 6]. From (2.13), it follows that

$$
\begin{aligned}
\left\|x_{n}-\bar{S} x_{n}\right\| & =\left\|\overline{S S}^{-1} x_{n}-\bar{S} x_{n}\right\| \\
& \leq\left\|\bar{S}^{-1} x_{n}-x_{n}\right\| \\
& =\left\|(2 I-S) x_{n}-x_{n}\right\|=\left\|x_{n}-S x_{n}\right\| \rightarrow 0 \quad(n \rightarrow \infty) .
\end{aligned}
$$

That is,

$$
\lim _{n \rightarrow \infty}\left\|x_{n}-\bar{S} x_{n}\right\|=0 .
$$

Next, we claim that $\limsup _{n \rightarrow \infty}\left\langle(\delta f-\mu F) z, x_{n}-z\right\rangle \leq 0$, where $z=P_{\Omega}(z-\mu F z+\delta f(z))$. For any $x, y \in H_{1}$, we conclude from Lemma 1.2 that

$$
\begin{aligned}
& \left\|P_{\Omega}(I-\mu F+\delta f) x-P_{\Omega}(I-\mu F+\delta f) y\right\| \\
& \leq \delta\|f(x)-f(y)\|+\|(I-\mu F) x-(I-\mu F) y\| \\
& \leq \delta\|x-y\|+(1-\tau)\|x-y\| \\
& =[1-(\tau-\delta)]\|x-y\|,
\end{aligned}
$$

which implies that $P_{\Omega}(I-\mu F+\delta f)$ is a contractive mapping. Banach's Contraction Mapping Principle tells us that $P_{\Omega}(I-\mu F+\delta f)$ has a unique fixed point, say $z \in H_{1}$, that is, $z=P_{\Omega}(z-\mu F z+\delta f(z))$. Since $\left\{x_{n}\right\}$ is a bounded sequence in $H_{1}$, without loss of generality, we may choose a subsequence $\left\{x_{n_{i}}\right\}$ of $\left\{x_{n}\right\}$ such that

$$
\limsup _{n \rightarrow \infty}\left\langle(\delta f-\mu F) z, x_{n}-z\right\rangle=\lim _{i \rightarrow \infty}\left\langle(\delta f-\mu F) z, x_{n_{i}}-z\right\rangle .
$$

Since $\left\{x_{n_{i}}\right\}$ is bounded, there exists a subsequence $\left\{x_{n_{i_{j}}}\right\}$ of $\left\{x_{n_{i}}\right\}$ which converges weakly to $w$. Without loss of generality, we may assume that $x_{n_{i}} \rightarrow w$. Thus, in terms of Lemma 1.6, (2.14) and the nonexpansivity of $\bar{S}$, we obtain that $w \in \operatorname{Fix}(\bar{S})=\operatorname{Fix}(S)=\bigcap_{n=1}^{\infty} \operatorname{Fix}\left(S_{n}\right)$. In addition, according to Lemma 1.6, (2.11) and the nonexpansivity of $G$, we have that $w \in \operatorname{Fix}(G)$ (due to Lemma 1.3). Consequently, we get $w \in \Omega$. Since $z=P_{\Omega}(z-\mu F z+\delta f(z))$ and $w \in \Omega$, we deduce that

$$
\begin{aligned}
\limsup _{n \rightarrow \infty}\left\langle(\delta f-\mu F) z, x_{n}-z\right\rangle & =\lim _{i \rightarrow \infty}\left\langle(\delta f-\mu F) z, x_{n_{i}}-z\right\rangle \\
& =\langle(\delta f-\mu F) z, w-z\rangle \\
& =\langle(z-\mu F z+\delta f(z))-z, w-z\rangle \\
& \leq 0
\end{aligned}
$$


Finally, we claim that $x_{n} \rightarrow z$ and $u_{n} \rightarrow z$ as $n \rightarrow \infty$. From Lemma 1.3, we have

$$
\begin{aligned}
\left\|x_{n+1}-z\right\|^{2} & =\alpha_{n}\left\langle(\delta f-\mu F) z, x_{n+1}-z\right\rangle+\beta_{n}\left\langle x_{n}-z, x_{n+1}-z\right\rangle \\
& +\left\langle\left[\left(1-\beta_{n}\right) I-\alpha_{n} \mu F\right] y_{n}-\left[\left(1-\beta_{n}\right) I-\alpha_{n} \mu F\right] z, x_{n+1}-z\right\rangle \\
& \leq \alpha_{n}\left\langle(\delta f-\mu F) z, x_{n+1}-z\right\rangle+\beta_{n}\left\langle x_{n}-z, x_{n+1}-z\right\rangle \\
& +\left(1-\beta_{n}-\alpha_{n} \tau\right)\left\|y_{n}-z\right\|\left\|x_{n+1}-z\right\| \\
& \leq \alpha_{n}\left\langle(\delta f-\mu F) z, x_{n+1}-z\right\rangle+\frac{1}{2} \beta_{n}\left(\left\|x_{n}-z\right\|^{2}+\left\|x_{n+1}-z\right\|^{2}\right) \\
& +\left(1-\beta_{n}-\alpha_{n} \tau\right)\left\|x_{n}-z\right\|\left\|x_{n+1}-z\right\| \\
& \leq \alpha_{n}\left\langle(\delta f-\mu F) z, x_{n+1}-z\right\rangle+\frac{1}{2}\left(1-\alpha_{n} \tau\right)\left(\left\|x_{n}-z\right\|^{2}+\left\|x_{n+1}-z\right\|^{2}\right) .
\end{aligned}
$$

This immediately implies that

$$
\begin{aligned}
2\left\|x_{n+1}-z\right\|^{2} & \leq 2 \alpha_{n}\left\langle(\delta f-\mu F) z, x_{n+1}-z\right\rangle+\left(1-\alpha_{n} \tau\right)\left(\left\|x_{n}-z\right\|^{2}+\left\|x_{n+1}-z\right\|^{2}\right) \\
& =2 \alpha_{n}\left\langle(\delta f-\mu F) z, x_{n+1}-z\right\rangle+\left(1-\alpha_{n} \tau\right)\left\|x_{n}-z\right\|^{2}+\left(1-\alpha_{n} \tau\right)\left\|x_{n+1}-z\right\|^{2} \\
& \leq 2 \alpha_{n}\left\langle(\delta f-\mu F) z, x_{n+1}-z\right\rangle+\left(1-\alpha_{n} \tau\right)\left\|x_{n}-z\right\|^{2}+\left\|x_{n+1}-z\right\|^{2},
\end{aligned}
$$

that is,

$$
\left\|x_{n+1}-z\right\|^{2} \leq\left(1-\alpha_{n} \tau\right)\left\|x_{n}-z\right\|^{2}+2 \alpha_{n}\left\langle(\delta f-\mu F) z, x_{n+1}-z\right\rangle .
$$

From condition (2), (3.32), and Lemma 1.7, we see that $\lim _{n \rightarrow \infty}\left\|x_{n}-z\right\|=0$. This completes the proof.

Remark 2.1. Comparing Theorem 2.1 with [18, Theorem 3.1], we have the following points. (1) Our algorithm, which is based on the Mann implicit iteration method, viscosity approximation method, and hybrid steepest-descent method, is more more subtle than algorithm 3.2 in [18, Theorem 3.1] because our algorithm involves the predictor-corrector for finding a common fixed point of a countable family of $\ell$-uniformly Lipschitzian pseudocontractive mappings $\left\{S_{i}\right\}_{i=1}^{\infty}$, that is, the implicit iterative step $u_{n}=$ $\gamma_{n} x_{n}+\left(1-\gamma_{n}\right) S_{n} u_{n}$ is the predictor one for finding their common fixed points. SVIP (1.2)-(1.3) with a HVI constraint for a countable family of nonexpansive mappings in Theorem [18, Theorem 3.1] is extended to a countable family of $\ell$-uniformly Lipschitzian pseudocontractive mappings, which is an essential difference between two HVI constraints.

\section{Acknowledgments}

The author is grateful to the anonymous referee for useful suggestions which improved the contents of this paper. This research was partially funded by supported by the Innovation Program of Shanghai Municipal Education Commission (15ZZ068), Ph.D. Program Foundation of Ministry of Education of China (20123127110002) and Program for Outstanding Academic Leaders in Shanghai City (15XD1503100).

\section{REFERENCES}

[1] C. Byrne, Iterative oblique projection onto convex sets and the split feasibity problem, Inverse Probl. 18 (2002), $441-453$.

[2] C. Byrne, Y. Censor, A. Gibali, S. Reich, The split common null point problem, J. Nonlinear Convex Anal. 13 (2012), 759-775.

[3] L.C. Ceng, Y.C. Lin, C.F. Wen, Iterative methods for triple hierarchical variational inequalities with mixed equilibrium problems, variational inclusions, and variational inequalities constraints, J. Inequal. Appl. 2015 (2015), Article ID 16.

[4] L.C. Ceng, M.M. Wong, J.C. Yao, A hybrid extragradient-like approximation method with regularization for solving split feasibility and fixed point problems, J. Nonlinear Convex Anal. 14 (2013), 163-182. 
[5] L.C. Ceng, N.C. Wong, J.C. Yao, Hybrid extragradient methods for finding minimum-norm solutions of split feasibility problems, J. Nonlinear Convex Anal. 16 (2015), 1965-1983.

[6] L.C. Ceng, Q.H. Ansari, J.C. Yao, Relaxed extragradient methods for finding minimum-norm solutions of the split feasibility problem, Nonlinear Anal. 75 (2012), 2116-2125.

[7] Y. Censor, T. Elfving, A multiprojection algorithm using Bregman projection in product space, Numer. Algorithms 8 (1994), 221-239.

[8] Y. Censor, T. Bortfeld, B. Martin, A. Trofimov, A unified approach for inversion problems in intensity-modulated radiation therapy, Phys. Med. Biol. 51 (2003), 2353-2365.

[9] Y. Censor, T. Elfving, N. Kopf, T. Bortfeld, The multiple-sets split feasibility problem and its applications for inverse problem, Inverse Probl. 21 (2005), 2071-2084.

[10] K. Deimling, Zeros of accretive operators, Manuscripta Math. 13 (1974), 365-374.

[11] N. Fang, Some results on split variational inclusion and fixed point problems in Hilbert spaces, Commun. Optim. Theory 2017 (2017), Article ID 5.

[12] K. Goebel, W.A. Kirk, Topics on Metric Fixed-Point Theory. In: Cambridge Studies in Advanced Mathematics, vol. 28, Cambridge University Press, 1990.

[13] H. Iiduka, Iterative algorithm for solving triple-hierarchical constrained optimization problem, J. Optim. Theory Appl. 148 (2011), 580-592.

[14] K.R. Kazmi, S.H. Rizvi, An iterative method for split variational inclusion problem and fixed point problem for a nonexpansive mapping, Optim. Lett. 8 (2014), 1113-1124.

[15] R.H. Martin Jr., Differential equations on closed subsets of a Banach space, Trans. Amer. Math. Soc. 179 (1973), $399-414$.

[16] A. Moudafi, Split monotone variational inclusions, J. Optim. Theory Appl. 150 (2011), 275-283.

[17] Z. Opial, Weak convergence of successive approximations for nonexpansive mappings, Bull. Amer. Math. Soc. 73 (1967), 591-597.

[18] K. Sitthithakerngkiet, J. Deepho, J. Martinez-Moreno, P. Kumam, Convergence analysis of a general iterative algorithm for finding a common solution of split variational inclusion and optimization problems, Numer. Algorithms 79 (2018), 801-824.

[19] T. Suzuki, Strong convergence of Krasnoselskii and Mann's type sequences for one-parameter nonexpansive semigroups without Bochner integrals, J. Math. Anal. Appl. 305 (2005), 227-239.

[20] W. Takahashi, C.F. Wen, J.C. Yao, An implicit algorithm for the split common fixed point problem in Hilbert spaces and applications, Appl. Anal. Optim. 1 (2017), 423-439.

[21] J. Wang, Y. Wang, Strong convergence of a cyclic iterative algorithm for split common fixed-point problems of demicontractive mappings, J. Nonlinear Var. Anal. 2 (2018), 295-303.

[22] H.K. Xu, T.H. Kim, Convergence of hybrid steepest-descent methods for variational inequalities, J. Optim. Theory Appl. 119 (2003), 185-201.

[23] Z. Xue, H. Zhou, Y.J. Cho, Iterative solutions of nonlinear equations for m-accretive operators in Banach spaces, J. Nonlinear Convex Anal. 1 (2000), 313-320. 